\title{
Serum hsa-miR-98-5p and RORC may be new biomarkers related to esophageal cancer
}

\author{
Zheyu Zhou ${ }^{1}$, Yinyu Wang ${ }^{1}$, Wenwen $\mathrm{Li}^{2}$, Haoyang Xia ${ }^{1}$, and Gaohua Han ${ }^{3, *}$ \\ ${ }^{1}$ School of Medicine \& Holistic Integrative Medicine, Nanjing University of Chinese Medicine, Nanjing 210023, China \\ ${ }^{2}$ School of pharmacy, Nanjing University of Chinese Medicine, Nanjing 210023, China \\ ${ }^{3}$ Department of Oncology, Taizhou People's Hospital, Taizhou 225300, China
}

\begin{abstract}
This study aims to use bioinformatics methods to discover new serum miRNA markers for esophageal cancer, and provide a theoretical basis for early diagnosis of esophageal cancer. We used GEO2R to analyze the differential serum miRNAs in esophageal cancer based on GSE112264 from the GEO database. Then target genes of top 10 differential miRNAs were predicted. Obtain RNA-Seq data of esophageal cancer from the TCGA database, and use R software for analysis of differential expression. Overlap the predicted target genes with the differentially down-regulated genes, then perform analysis of GO and KEGG enrichment. Use GEPIA and UALCAN databases to perform verification of expression and prognostic analysis of key genes in the pathway. The results showed there are 2565 differential miRNAs in the serum of esophageal cancer patients. The top 10 up-regulated miRNAs predicted 1676 target genes, then 63 overlapped genes were obtained from target genes and 1642 down-regulated genes. GO enrichment obtained 14 biological processes, and KEGG enrichment obtained the circadian rhythm pathway. Only RORC is related to the poor prognosis of patients with esophageal cancer. Our study concluded serum hsa-miR-98-5p and its target gene RORC may be new biological markers for early diagnosis and treatment of esophageal cancer.
\end{abstract}

\section{Introduction}

Cancer has become the leading cause of death in China. Based on the latest data from the National Cancer Center, the incidence of esophageal cancer ranks the sixth among all malignant tumors and the fourth among digestive system tumors ${ }^{[1]}$. GLOBOCAN estimated in 2018 that the occurrence and death of esophageal cancer in China accounted for $53.7 \%$ and $55.7 \%$ of the world's esophageal cancer cases, respectively ${ }^{[2]}$. Although the actual number of cases and deaths of esophageal cancer is lower than GLOBOCAN's estimate ${ }^{[1]}$, its poor prognosis is still an important cancer burden. Therefore, the discovery of new biomarkers for esophageal cancer is of great significance to provide evidence for the diagnosis and treatment of esophageal cancer.

Transcripts include various types of RNA molecules, such as microRNA (miRNA), long non-coding RNA (lncRNA), circular RNA (circRNA) and messenger RNA $(\text { mRNA })^{[3]}$. MiRNA is a small non-coding RNA with a length of about 22 nucleotides, which has the ability to regulate the expression of target genes by inhibiting translation of mRNAs or promoting degradation of mRNAs at the post-transcriptional level ${ }^{[4]}$. Studies have shown that miRNAs play an important role in the occurrence and development of a variety of cancers. In esophageal cancer, miR-373 and miR-26b can enhance proliferation and migration of cells, while miR-100 can inhibit proliferation, migration and invasion of cells. MiR-98 can restore the sensitivity of cells to radiotherapy, and at the same time, miR-124 can increase apoptosis of cells after radiotherapy ${ }^{[5]}$. Therefore, miRNA is expected to become a new target for the treatment of esophageal cancer.

This article uses bioinformatics methods to analyze the differential serum circulating miRNAs and differentially expressed mRNAs of esophageal cancer in various databases (Fig.1 shows workflow of this work). We hope to provide a new theoretical basis for clinical diagnosis and treatment of esophageal cancer by exploring the key relationship of miRNA-mRNA regulatory.

\footnotetext{
*Corresponding author: danny_75@njmu.edu.cn
} 


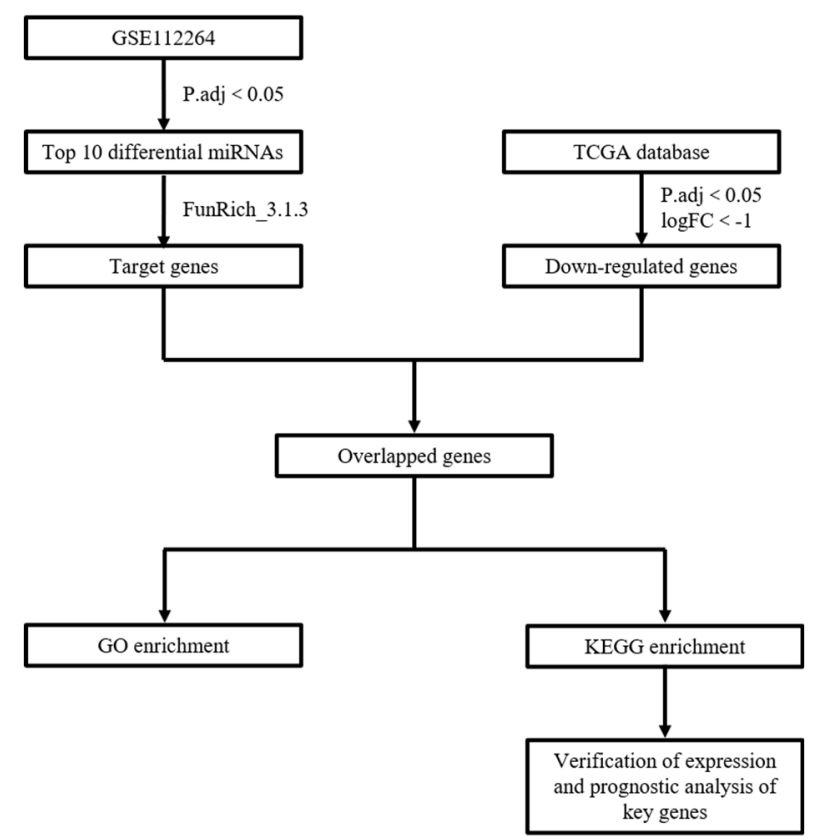

Fig.1 Workflow of this study

\section{Materials and methods}

\subsection{Acquisition and analysis of data}

Search the GSE112264 data set in the GEO database (https://www.ncbi.nlm.nih.gov/geo/), and use the GEO2R online analysis software to analyze the difference between the serum circulating miRNAs of 50 esophageal cancer and 41 normal control samples in the data set (Padj $<0.05$ was used as cut-off criteria). Download RNA-Seq data of esophageal cancer in the TCGA database (https://portal.gdc.cancer.gov), which include 160 esophageal cancers and 11 normal control samples. Use $\mathrm{R}$ software and quote the 'limma' package for differential analysis $(\operatorname{Padj}<0.05$ and $|\operatorname{logFC}|>1$ were used as the cut-off criteria).

\subsection{Prediction of target genes for differential miRNAs}

Use Microsoft Excel to sort the differential miRNAs in descending order by $|\log \mathrm{FC}|$, and use the FunRich_3.1.3 software to predict the target genes of the top 10 miRNAs.

\subsection{Screening and enrichment analysis of overlapped genes}

Use R software and quote the 'venn' package to obtain overlapped genes between target genes predicted by miRNAs and differentially down-regulated genes in the TCGA database. Use the 'BiocManager' package to perform analysis of GO and KEGG enrichment on the obtained overlapped genes.

\subsection{Verification and analysis of key genes in the KEGG pathway}

Use GEPIA database (https://gepia.cancer-pku.cn) to verify the expression of key genes in the KEGG pathway, and use UALCAN database (https://ualcan.path.uab.edu) to evaluate the overall survival rate and expression of key genes.

\section{Results}

\subsection{Acquisition of differential miRNAs and mRNAs}

2565 differential miRNAs in serum were obtained by analysis of the GSE112264 data set. The first 10 differential miRNAs are all significantly up-regulated (Table 1). After analyzing the RNA-Seq data of esophageal cancer in the TCGA database, 5386 differentially expressed mRNAs were obtained, of which 1642 were down-regulated.

Table 1 Top 10 differential miRNAs

\begin{tabular}{lll}
\hline miRNA_ID_LIST & $\log F \mathrm{C}$ & adj.P.Val \\
\hline hsa-miR-1246 & 6.484617 & $5.28 \mathrm{E}-15$ \\
hsa-miR-191-5p & 6.075684 & $4.24 \mathrm{E}-18$ \\
hsa-miR-5692b & 5.927485 & $4.18 \mathrm{E}-18$ \\
hsa-miR-3688-5p & 5.338556 & $1.01 \mathrm{E}-15$ \\
hsa-miR-98-5p & 5.065285 & $1.93 \mathrm{E}-14$ \\
hsa-miR-24-1-5p & 5.023674 & $4.90 \mathrm{E}-13$ \\
hsa-miR-142-3p & 4.925972 & $3.01 \mathrm{E}-13$ \\
hsa-miR-99a-5p & 4.870421 & $8.80 \mathrm{E}-12$ \\
hsa-miR-16-5p & 4.869812 & $6.64 \mathrm{E}-14$ \\
hsa-miR-19a-5p & 4.81999 & $1.44 \mathrm{E}-12$ \\
\hline
\end{tabular}

\subsection{Prediction of target genes for differential miRNAs}

None target genes were predicted by 5 miRNAs (hsa-miR-1246, hsa-miR-5692b, hsa-miR-3688-5p, hsa-miR-24-1-5p and hsa-miR-19a-5p) in table 1, the remaining 5 miRNAs (hsa-miR-191-5p, hsa-miR-98-5p, hsa-miR-142-3p, hsa-miR-99a-5p and hsa-miR-16-5p) predicted 1676 target genes in total.

\subsection{Screening and enrichment analysis of overlapped genes}

Due to the significant up-regulation of miRNA, the target genes regulated by it should be down-regulated significantly. A total of 63 overlapped genes were obtained from 1676 target genes predicted by miRNAs and 1642 differentially down-regulated genes in esophageal cancer (Fig.2). Analysis of GO enrichment $(\mathrm{P}<0.05)$ obtained 14 biological processes, and table 2 lists the first 5 results of enrichment. Analysis of KEGG enrichment $(\mathrm{P}<0.05)$ obtained only one pathway (Table 
3). There are three genes (RORC/PRKAA2/CRY2) that play a role in this pathway, suggesting that these three genes may be key genes. At the same time, these three genes are all target genes of hsa-miR-98-5p.

Down-regulated genes Target genes

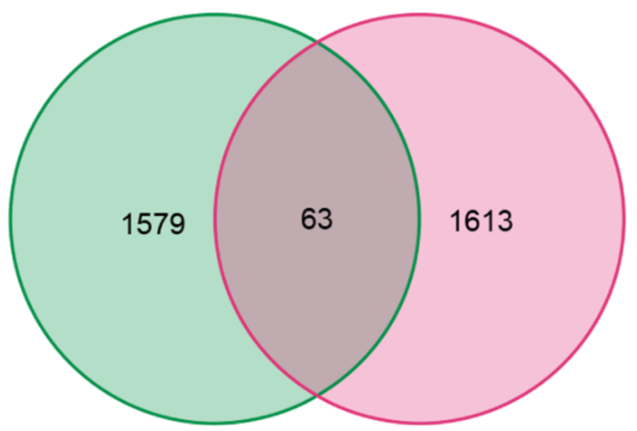

Fig.2 Venn diagram of overlapped genes

Table 2 Top 5 results of GO enrichment

\begin{tabular}{lllll}
\hline Ontology & ID & Description & GeneRatio & $\mathrm{p}$-Value \\
\hline BP & GO:0001101 & response to acid chemical & $9 / 58$ & $1.04 \mathrm{E}-06$ \\
BP & GO:0007613 & memory & $5 / 58$ & $3.12 \mathrm{E}-05$ \\
BP & GO:0048511 & rhythmic process & $7 / 58$ & $3.45 \mathrm{E}-05$ \\
BP & GO:0071229 & cellular response to acid chemical & $6 / 58$ & $4.56 \mathrm{E}-05$ \\
BP & GO:0009755 & hormonc-mediatcd signaling pathway & $6 / 58$ & $8.34 \mathrm{E}-05$ \\
\hline
\end{tabular}

Table 3 Results of KEGG pathway enrichment

\subsection{Verification and analysis of RORC/PRKAA2/CRY2}

The GEPIA database includes 182 esophageal cancers and 286 normal control samples. The expression of RORC/PRKAA2/CRY2 were verified, and it was found that only the expression of RORC was consistent with the TCGA database (Fig.3). Whether the expression of RORC/PRKAA2/CRY2 influences the overall survival rate of esophageal cancer patients were analyzed based on the UALCAN database, it was found that only low expression of RORC significantly reduced the overall survival rate of esophageal cancer patients (Fig.4). The above results suggested that the target gene RORC of hsa-miR-98-5p may play an important role in esophageal cancer. Finally, the UALCAN database was used again to analyze the expression of RORC, and it was found that the expression of RORC was down-regulated in both esophageal adenocarcinoma and esophageal squamous cell carcinoma (ESCC). At the same time, with the increase in tumor pathological stage, the expression of RORC also has a downward trend (Fig.5).

\begin{tabular}{lllll}
\hline Pathway ID & Description & GencRatio & p-Valuc & Gcncs \\
\hline hsa04710 & Circadian rhythm & $3 / 34$ & 0.000279917 & RORC/PRKAA2/CRY2 \\
\hline
\end{tabular}
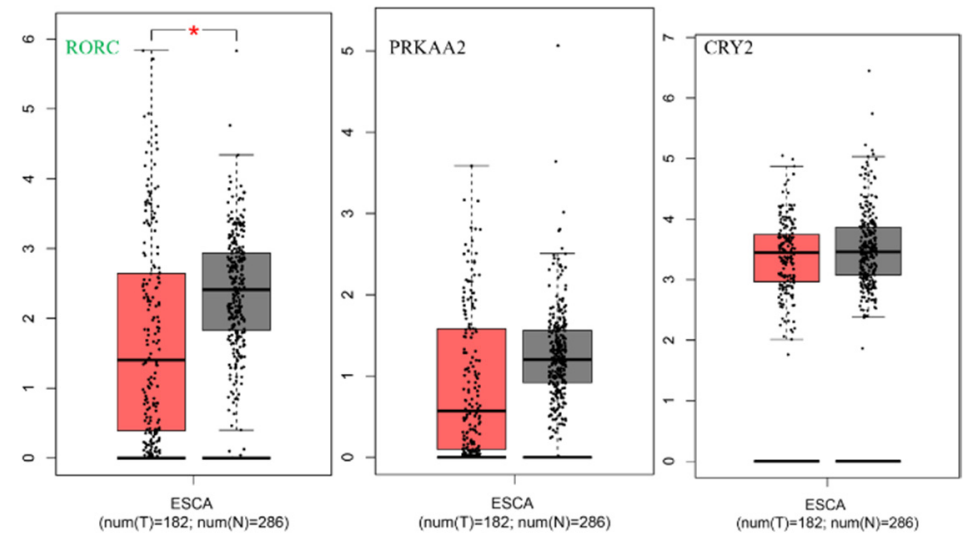

Fig.3 Verification of RORC/PRKAA2/CRY2 expression in GEPIA
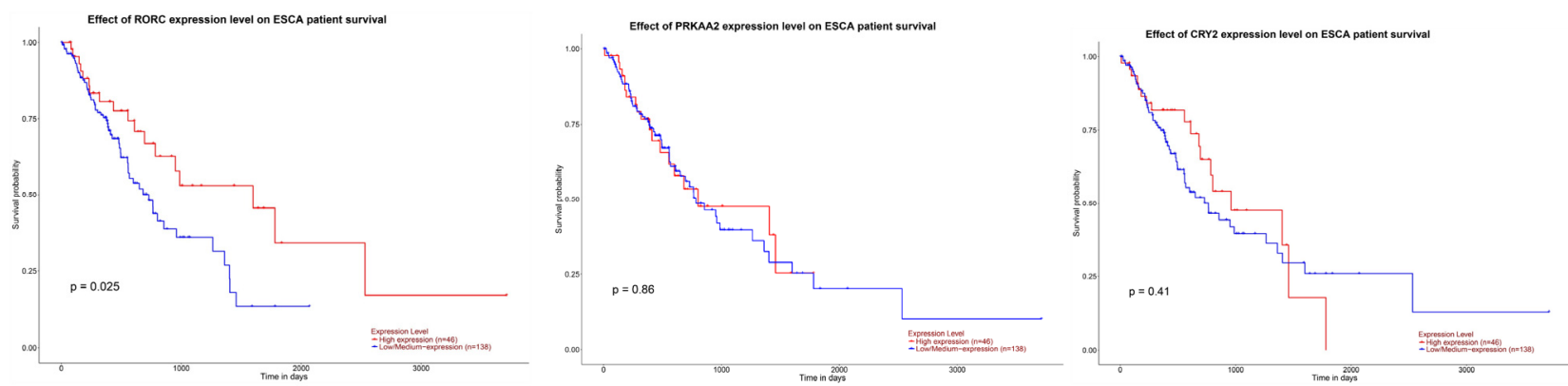

Fig.4 The effect of RORC/PRKAA2/CRY2 on the survival rate of patients with esophageal cancer 


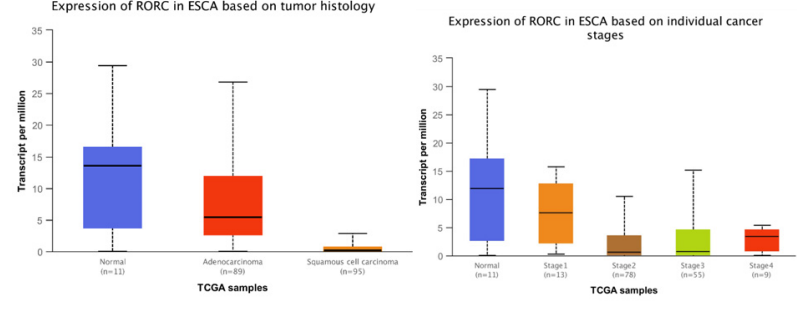

Fig.5 Verification of RORC expression in UALCAN

\section{Discussion}

Early stage of esophageal cancer lacks specific symptoms and is easy to be ignored by patients. It is usually at an advanced stage at the time of treatment, so the effect of treatment and the prognosis of advanced esophageal cancer are poor ${ }^{[6]}$. So far, there is still a lack of effective biological markers in the early diagnosis and prognosis of esophageal cancer. For example, carcinoembryonic antigen (CEA) and squamous cell carcinoma antigen (SCC), these conventional markers lack sufficient sensitivity and specificity ${ }^{[7]}$. Therefore, there is an urgent need to discover new non-invasive biomarkers for the early diagnosis of esophageal cancer.

After comparing the serum miRNA profiles of patients with diffuse large B-cell lymphoma, C.H. Lawrie et al. first reported the potential of circulating miRNAs as tumor non-invasive specific biomarkers ${ }^{[8]}$. Unexpectedly, compared with serum mRNAs, serum miRNAs are more stable. Serum miRNAs are not only resistant to endogenous ribonuclease, but also resistant to conditions such as high/low $\mathrm{pH}$, boiling, freezing, and long-term storage. This indicates that serum miRNAs are reliable indicators for cancer detection ${ }^{[9,10]}$.

C. Zhang et al. reported that 7 serum miRNAs (miR-10a, miR-22, miR-100, miR-148b, miR-223, miR-133a and miR-127-3p) were significantly up-regulated in the serum of ESCC patients compared with the control group ${ }^{[11]}$. S. Komatsu et al. found that the expression ratio of miR-21/miR-375 in plasma can distinguish ESCC patients from healthy people. The sensitivity of this indicator is as high as $88 \%$, and the specificity is as high as $70 \%^{[12]}$. In addition, the serum concentration of miR-21 of ESCC patients is 8.4-fold higher than healthy controls, which is also considered to be related to tumor metastasis ${ }^{[13]}$. One month after surgical removal of the tumor, the serum concentration of miR-21 of ESCC patients was significantly reduced ${ }^{[12,14]}$.

This study found that hsa-miR-98-5p was significantly up-regulated in the serum of patients with esophageal cancer compared with healthy controls. There is no research reporting that hsa-miR-98-5p is related to esophageal cancer. In other cancers, D.H. Zhou et al. found that after transfection of hsa-miR-98-5p inhibitor, the survival rate of A549 and NCI-H460 cell lines was decreased upon treatment with cisplatin. As a key regulator of cisplatin-induced apoptosis, p53 is also negatively regulated by hsa-miR-98-5p. Inhibiting hsa-miR-98-5p can increase the expression of $\mathrm{p} 53^{[15]}$. D. Sun et al. found that hsa-miR-98-5p was significantly up-regulated in breast cancer cells through bioinformatics analysis, which was also verified by experiments in vitro ${ }^{[16]}$. Y. Wang et al. found that hsa-miR-98-5p contributed to resistance of cisplatin in epithelial ovarian cancer by suppressing biogenesis of miR-152 via targeting Dicer1 ${ }^{[17]}$.

The circadian rhythm pathway enriched by the target genes (RORC/PRKAA2/CRY2) of hsa-miR-98-5p is related to the regulation of circadian rhythm. S. Mocellin et al. found that the dysfunction of the biological clock and the genetic variation of the circadian rhythm pathway have a statistically significant correlation with breast cancer, prostate cancer and lung cancer. CRY2 is associated with the risk of breast cancer and lung cancer, while RORC is associated with the risk of breast cancer $^{[18]}$. After analyzing the role of 14 core genes of biological clock such as RORC/CRY2 in a series of cancers, Y. Wu et al. found that the four core negative regulatory genes such as CRY2 were always down-regulated, which also proved that the biological clock of cancer was destroyed. Abnormal biological clock depletes $\mathrm{T}$ cells and upregulates immunosuppressive molecules (such as PD-L1 and CTLA-4) at the same time. Therefore, the disorder of circadian rhythm is significantly related to the prognosis of patients ${ }^{[19]}$. Although PRKAA2 and CRY2 have not been found to be associated with the poor prognosis of patients with esophageal cancer, RORC is significantly down-regulated in esophageal cancer and related to the poor prognosis of patients is another finding of this study. There is no research reporting that RORC is related to esophageal cancer. Retinoic acid-related orphan receptor $\mathrm{C}$ (RORC) is a member of the nuclear orphan receptor family and plays a key regulatory role in the proliferation, metastasis and chemoresistance of various types of malignant tumors. D. Cao et al. revealed that the expression of RORC in the tumor tissues of patients with bladder cancer is lost. Enhanced expression of RORC can inhibit proliferation of cell and glucose metabolism, and increase cisplatin-induced apoptosis ${ }^{[20]}$. J. Wang et al. analyzed two data sets in the GEO database and found that the expression of RORC in metastatic prostate cancer was significantly higher than that of local prostate cancer and benign prostate tissue. At the same time, ROR $-\gamma$ is the encoded product of RORC. Immunohistochemistry shows that compared with benign prostate tissue, ROR- $\gamma$ is overexpressed in more than 50\% of tumors, and this is significantly related to metastasis of tumor ${ }^{[21]}$.

Therefore, hsa-miR-98-5p and its target gene RORC may also play a role in promoting the proliferation, metastasis, glucose metabolism and inducing drug resistance of esophageal cancer, which needs to be further verified by experiments.

\section{Conclusion}

In this paper, a new serum miRNA (hsa-miR-98-5p) related to esophageal cancer was discovered through bioinformatics methods. Its target gene RORC may affect the occurrence, development and prognosis of esophageal cancer by regulating the circadian rhythm. 
This provides a new serum biomarker for the early diagnosis of esophageal cancer, and also provides new potential targets for the treatment of esophageal cancer.

\section{Funding}

This study was supported by the Top-notch health talents project of 'Six-One Project' in Jiangsu Province (Grant No. LGY2019038) and Jiangsu Medical Innovation Team Project (Grant No. CXTDA2017042).

\section{References}

1. S. Zhang, K. Sun, R. Zheng, et al, Journal of the National Cancer Center (2020)

2. F. Bray, J. Ferlay, I. Soerjomataram, et al, CA Cancer J Clin, 68, 394-424 (2018)

3. Y. Tay, J. Rinn, P.P. Pandolfi, Nature, 505, 344-352 (2014)

4. D.S.M. Correia, M. Gjorgjieva, D. Dolicka, et al, Int J Mol Sci, 20 (2019)

5. X. Hou, J. Wen, Z. Ren, et al, Oncotarget, 8, 43571-43578 (2017)

6. P. Sharma, R. Sharma, Crit Rev Oncol Hematol, 96, 449-462 (2015)

7. S. Kosugi, T. Nishimaki, T. Kanda, et al, World J Surg, 28, 680-685 (2004)

8. C.H. Lawrie, S. Gal, H.M. Dunlop, et al, Br J Haematol, 141, 672-675 (2008)
9. X. Chen, Y. Ba, L. Ma, et al, Cell Res, 18, 997-1006 (2008)

10. P.S. Mitchell, R.K. Parkin, E.M. Kroh, et al, Proc Natl Acad Sci U S A, 105, 10513-10518 (2008)

11. C. Zhang, C. Wang, X. Chen, et al, Clin Chem, 56, 1871-1879 (2010)

12. S. Komatsu, D. Ichikawa, H. Takeshita, et al, Br J Cancer, 105, 104-111 (2011)

13. E.H. Cai, Y.X. Gao, Z.Z. Wei, et al, Asian Pac J Cancer Prev, 13, 1563-1567 (2012)

14. J. Kurashige, H. Kamohara, M. Watanabe, et al, J Surg Oncol, 106, 188-192 (2012)

15. D.H. Zhou, X. Wang, Q. Feng, Nutr Cancer, 66, 636-644 (2014)

16. D. Sun, X. Luo, L. Ma, et al, Life Sci, 261, 118435 (2020)

17. Y. Wang, W. Bao, Y. Liu, et al, Cell Death Dis, 9, 447 (2018)

18. S. Mocellin, S. Tropea, C. Benna, et al, BMC Med, 16, 20 (2018)

19. Y. Wu, B. Tao, T. Zhang, et al, Front Immunol, 10, 2451 (2019)

20. D. Cao, Z. Qi, Y. Pang, et al, Cancer Res, 79, 2604-2618 (2019)

21. J. Wang, J.X. Zou, X. Xue, et al, Nat Med, 22, 488-496 (2016) 